\title{
Evaluation of the Effects of Photodynamic Therapy Alone and Combined with Standard Antifungal Therapy on Planktonic Cells and Biofilms of Fusarium spp. and Exophiala spp.
}

OPEN ACCESS

Edited by:

Octavio Luiz Franco, Universidade Católica de Brasilia,

Brazil

Reviewed by:

Nuno C. Santos,

Faculdade de Medicina da Universidade de Lisboa, Portugal

Fabian Cieplik,

University Medical Center

Regensburg, Germany

*Correspondence:

Lujuan Gao

gao_lujuan@163.com:

Yisun

jzzxyysy@163.com

${ }^{\dagger}$ These authors have contributed equally to this work and are joint first authors.

Specialty section:

This article was submitted to

Antimicrobials, Resistance and

Chemotherapy,

a section of the journal

Frontiers in Microbiology

Received: 07 March 2016

Accepted: 14 April 2016

Published: 27 April 2016

Citation:

Gao L, Jiang S, Sun Y, Deng $M, W u Q$, Li M and Zeng T (2016) Evaluation of the Effects of Photodynamic Therapy Alone and Combined with Standard Antifungal Therapy on Planktonic Cells and Biofilms of Fusarium spp. and Exophiala spp.

Front. Microbiol. 7:617.

doi: 10.3389/fmicb.2016.00617

\section{Lujuan Gao ${ }^{1 * t}$, Shaojie Jiang ${ }^{2 \dagger}$, Yi Sun ${ }^{3 *}$, Meiqi Deng ${ }^{4}$, Qingzhi Wu ${ }^{3}, \mathrm{Ming}_{\mathrm{Li}}{ }^{1}$ and Tongxiang Zeng ${ }^{3}$}

${ }^{1}$ Department of Dermatology, Zhongshan Hospital Fudan University, Shanghai, China, ${ }^{2}$ Department of Gastroenterology, Jingzhou Central Hospital, The Second Clinical Medical College, Yangtze University, Jingzhou, China, ${ }^{3}$ Department of Dermatology, Jingzhou Central Hospital, The Second Clinical Medical College, Yangtze University, Jingzhou, China, ${ }^{4}$ The Second Clinical Medical College, Yangtze University, Jingzhou, China

Infections of Fusarium spp. and Exophiala spp. are often chronic, recalcitrant, resulting in significant morbidity, causing discomfort, disfigurement, social isolation. Systemic disseminations happen in compromised patients, which are often refractory to available antifungal therapies and thereby lead to death. The antimicrobial photodynamic therapy (APDT) has been demonstrated to effectively inactivate multiple pathogenic fungi and is considered as a promising alternative treatment for mycoses. In the present study, we applied methylene blue $(8,16$, and $32 \mu \mathrm{g} / \mathrm{ml})$ as a photosensitizing agent and light emitting diode $\left(635 \pm 10 \mathrm{~nm}, 12\right.$ and $\left.24 \mathrm{~J} / \mathrm{cm}^{2}\right)$, and evaluated the effects of photodynamic inactivation on five strains of Fusarium spp. and five strains of Exophiala spp., as well as photodynamic effects on in vitro susceptibility to itraconazole, voriconazole, posaconazole and amphotericin B, both planktonic and biofilm forms. Photodynamic therapy was efficient in reducing the growth of all strains tested, exhibiting colony forming unit-reductions of up to $6.4 \log _{10}$ and $5.6 \log _{10}$ against planktonic cultures and biofilms, respectively. However, biofilms were less sensitive since the irradiation time was twice longer than that of planktonic cultures. Notably, the photodynamic effects against Fusarium strains with high minimal inhibitory concentration (MIC) values of $\geq 16,4-8,4-8$, and $2-4 \mu \mathrm{g} / \mathrm{ml}$ for itraconazole, voriconazole, posaconazole and amphotericin B, respectively, were comparable or even superior to Exophiala spp., despite Exophiala spp. showed relatively better antifungal susceptibility profile. MIC ranges against planktonic cells of both species were up to 64 times lower after aPDT treatment. Biofilms of both species showed high sessile MIC50 (SMIC50) and SMIC80 of $\geq 16 \mu \mathrm{g} / \mathrm{ml}$ for all azoles tested and variable susceptibilities to amphotericin B, with SMIC ranging between 1 and $16 \mu \mathrm{g} / \mathrm{ml}$. Biofilms subjected to aPDT exhibited a distinct reduction in SMIC50 and SMIC80 compared to untreated groups for both species, except SMIC80 of itraconazole against Fusarium biofilms. In conclusion, in vitro photodynamic therapy was efficient in inactivation of Fusarium spp. and Exophiala 
spp., both planktonic cultures and biofilms. In addition, the combination of aPDT and antifungal drugs represents an attractive alternative to the current antifungal strategies. However, further investigations are warranted for the reliable and safe application in clinical practice.

Keywords: photodynamic inactivation, Fusarium, Exophiala, planktonic, biofilm, antifungal susceptibility

\section{INTRODUCTION}

Opportunistic fungi may causes cutaneous, subcutaneous and serious invasive infections, especially in immunocompromised and debilitated individuals. Invasive fungal infection represents a growing threat for human health due to difficulty in diagnosis and relatively few classes of available antifungal agents. Fusarium spp. and Exophiala spp. are both ubiquitous fungi commonly found in soil and on plants, and are increasingly recognized pathogen (Li et al., 2011; Guarro, 2013). $E$. dermatitidis is the leading cause of severe neurotropic phaeohyphomycosis (Li et al., 2011) and common cause of chromoblastomycosis; while fusariosis is, after aspergillosis, the second most common mold infection in humans, among which $F$. solani species complex and $F$. oxysporum species complex are responsible for approximately 60 and $20 \%$ of the cases, respectively (Guarro, 2013). Human infection usually occurs as a result of inoculation of the organism through the body surface causing local infection. Systemic dissemination, whose prevalence is effectively growing, occurs in subjects with underlying immunodeficiency (Li et al., 2011; Guarro, 2013), which is often refractory to available antifungal therapies and thereby leads to death (Filizzola et al., 2003; Guarro, 2013). Early management of local infection is crucial to prevent lifethreatening disease. However, available antifungal drugs have shown poor in vitro activity against Fusarium spp. (Guarro, 2013). Fusariosis is mostly refractory to treatment, with a high mortality rate for systemic disseminations (Guarro, 2013). As for Exophiala spp. infection, success rate was only $40-70 \%$ although favorable in vitro activity of most antifungal drugs has been shown (Revankar and Sutton, 2010; Kondori et al., 2011; Patel et al., 2013). In addition to life-threatening infection, more frequently they result in significant morbidity, causing discomfort, disfigurement, social isolation, and they are usually recurrent and chronic.

Biofilm formation, which is a prerequisite event toward the development of invasive disease, has been reported to involve in about $80 \%$ of non-acute infections in human (Davies, 2003). Fungal biofilms, unlike planktonic forms, are relatively resistant to conventional antifungals, which may play an important role in the dissemination and therapeutic failure of Fusaruim and Exophiala infections (Desai et al., 2014). However, the eradication of biofilms is still a key challenge in the antifungal discovery agenda. Given that fungal infections always require lengthy antifungal therapy, alternative treatment methods are urgently needed.

In recent years, due to its efficiency and low invasive character, antimicrobial photodynamic therapy (aPDT) arises as a promising alternative approach to conventional antifungal medications, which has been demonstrated effective against multiple pathogenic fungi in vitro (Lyon et al., 2013; Pires et al., 2013; Arboleda et al., 2014; Morton et al., 2014; Nunes Mario et al., 2014; Baltazar et al., 2015) and applied successfully in vivo against cutaneous and subcutaneous mycoses (Gilaberte et al., 2011; Lyon et al., 2011b; Souza et al., 2014). In addition, aPDT could be a useful approach for the control of biofilms, and has been proposed for the growth control of oral candida (Pereira et al., 2011; Pereira Gonzales and Maisch, 2012). In vitro studies have shown that aPDT with methylene blue and light emitting diode (LED) was highly effective in killing Foncecaea pedrosoi, Cladophialophora pedrosoi, Sporothrix schenckii complex species et al. (Lyon et al., 2013; Nunes Mario et al., 2014). Lyon JP et al. employed methylene blue as photosensitizer and a LED device as light source, denoting the efficacy of aPDT in vivo against chromoblastomycosis (Lyon et al., 2011b). In this trial, an improvement of $90 \%$ of the clinical and histological aspect of the lesions was observed in all the 10 patients (Lyon et al., 2011b). Gilaberte et al. reported aPDT with $16 \%$ methylaminolevulinate cream and $635 \mathrm{~nm}$ LED successfully treated onychomycosis caused by the F. oxysporum, which was unresponsive to standard antifungals (Gilaberte et al., 2011). Despite these encouraging findings, aPDT revealed different effects against Fusarium spp. Rose bengal-mediated aPDT has been demonstrated to successfully inhibit the growth of $F$. solani (Arboleda et al., 2014). Pre-exposure to amphotericin B allowed riboflavin combined with long-wave ultraviolet effectiveness against Fusarium spp. (Sauer et al., 2010), while the combination of riboflavin and longwave ultraviolet alone showed no antifungal effect on F. solani (Kashiwabuchi et al., 2013). In addition, little is known regarding the in vitro effects of aPDT on the growth and antifungal susceptibility of Fusarium spp. and the melanized pathogen Exophiala spp.

The aim of this study was to evaluate the effects of aPDT mediated by methylene blue with combination of LED on the viability of in vitro planktonic and biofilm forms of Fusaruim spp. and Exophiala spp., and to investigate the effects of aPDT on the antifungal susceptibilities.

\section{MATERIALS AND METHODS}

\section{Fungal Strains}

Five strains of E. dermatitidis and 5 strains of Fusarium spp. (3 strains of F. solani, and 2 strains of F. oxysporum) were studied. All strains were clinical isolates and identified by molecular and morphologic methods. For the dermtermiantion of in vitro susceptibilities against antifungal agents, Candida parapsilosis ATCC 22019 was included to ensure quality control. 


\section{Antifungal Agents}

All antifungal drugs including itraconazole (ITC; purity $\geq 99 \%$ ), voriconazole (VRC; purity $\geq 99 \%$ ), posaconazole (POS; purity $\geq$ 99\%), and amphotericin (AMB; purity $\geq 80 \%$ ) were purchased in powder form from Sigma Chemical Co., St. Louis, MO and prepared as outlined in the Clinical and Laboratory Standards Institute (CLSI) broth microdilution method M38-A2 (Institute, 2008). The working concentration ranges of tested drugs were all $0.06-16 \mu \mathrm{g} / \mathrm{ml}$.

\section{Inoculum Preparation}

Conidia harvested from cultures grown for 7 days on Sabouraud dextrose agar (SDA) were suspended in sterile distilled water containing $0.03 \%$ Triton and diluted to a concentration of 1 $5 \times 10^{6}$ spores $/ \mathrm{ml}$. One milliliter of the suspension was added into $100 \mathrm{ml}$ Sabouraud Dextrose broth. After incubation at $27^{\circ} \mathrm{C}$ in a shaker at $180 \mathrm{rpm}$ for $48 \mathrm{~h}$, conidia were collected and resuspended in saline solution to obtain a suspension of $1-5 \times$ $10^{6} \mathrm{spores} / \mathrm{ml}$.

\section{Biofilm Preparation}

Conidia were collected from SDA and resuspended in $20 \mathrm{ml}$ Roswell Park Memorial Institute-1640 (RPMI-1640). RPMI-1640 without sodium bicarbonate supplemented with L-glutamine (Cellgro, cat. no. 50-020-PB) and buffered with $165 \mathrm{mM}$ morpholinepropanesulfonic acid (Fisher, cat. no. BP308) to $\mathrm{pH}$ 7 is used for biofilm preparation according to the protocol (Pierce et al., 2008). The suspension was then adjusted to the final concentration of $1 \times 10^{7}$ spores $/ \mathrm{ml}$. Subsequently, the suspension was added into the 96-well plate with $200 \mu \mathrm{l}$ in each cell and incubated at $37^{\circ} \mathrm{C}$ for 48 and $72 \mathrm{~h}$ for Fusarium spp. and Exophiala spp., respectively. The media were then carefully extracted without disturbing the biofilm. The 96-well plate was washed with sterile PBS for three times to remove detached spores (Pierce et al., 2008).

\section{Photodynamic Therapy of Planktonic Cultures}

The photodynamic inactivation technique was described by Lyon et al. (2013), with modifications in the volume used, the incubation time, and the concentrations of methylene blue. The methylene blue was tested at concentrations of $8 \mu \mathrm{g} / \mathrm{ml}$ (T1), $16 \mu \mathrm{g} / \mathrm{ml}$ (T2), and $32 \mu \mathrm{g} / \mathrm{ml}$ (T3), with $100 \mu \mathrm{l}$ of each concentration mixed with $100 \mu \mathrm{l}$ of the standardized inocula prealiquoted into sterile 96 -well microtiter plates. The suspensions were incubated for $2 \mathrm{~h}$ in the dark at $37^{\circ} \mathrm{C}$. After this period, the inocula were irradiated using a LED with an irradiance of $100 \mathrm{~mW} / \mathrm{cm}^{2}$ at a wavelength of $635 \pm 10 \mathrm{~nm}$ and at a distance of $1 \mathrm{~cm}$ for $120 \mathrm{~s}\left(12 \mathrm{~J} / \mathrm{cm}^{2}\right)$. The following controls were included: fungal suspension in saline without irradiation (C1), fungal suspension with methylene blue $(16 \mu \mathrm{g} / \mathrm{ml})$ and without irradiation (C2), and fungal suspension in saline and irradiated (C3). After the irradiation period, $10 \mu \mathrm{l}$ aliquots from each group were suspended with $90 \mu \mathrm{l}$ saline, subsequently inoculated on SDA and incubated at $37^{\circ} \mathrm{C}$ for 48 and $120 \mathrm{~h}$ for Fusarium spp. and Exophiala spp., respectively. The viability of the conidia was then determined by counting colony-forming unit (CFU). All tests were performed in triplicate.

\section{Photodynamic Therapy of Biofilms}

The methylene blue was also tested at concentrations as above, with $100 \mu \mathrm{l}$ of each concentration added into 96-well plates containing biofilms. After incubation in dark for $2 \mathrm{~h}$ at $37^{\circ} \mathrm{C}$, the biofilms were irradiated using a LED with an irradiance of 100 $\mathrm{mW} / \mathrm{cm}^{2}$ at a wavelength of $635 \pm 10 \mathrm{~nm}$ and at a distance of 1 $\mathrm{cm}$ for $240 \mathrm{~s}\left(24 \mathrm{~J} / \mathrm{cm}^{2}\right)$. The controls were included: biofilms in saline without irradiation (C1), biofilms with methylene blue (16 $\mu \mathrm{g} / \mathrm{ml}$ ) and without irradiation (C2), and biofilms in saline and irradiated (C3). After PDT treatment, $100 \mu \mathrm{l}$ of sterile water was added into the well and washed vigorously in order to resuspend the biofilm cells thoroughly. The suspensions were then diluted 1000 times in sterile water and $100 \mu \mathrm{l}$ aliquots were inoculated evenly on SDA. The number of CFU was determined after 24 and $48 \mathrm{~h}$ incubation at $37^{\circ} \mathrm{C}$ for Fusarium spp. and Exophiala spp., respectively. All tests were performed in triplicate.

\section{In vitro Antifungal Susceptibility of Planktonic Cultures}

The individual minimal inhibitory concentrations (MICs) of ITC, VRC, POS, and AMB on photodynamic treated (T1) and untreated planktonic cells were determined according to M38A2 method (Institute, 2008). The 96-well plate was inoculated with $100 \mu \mathrm{l}$ of the inoculum suspension prepared and $100 \mu \mathrm{l}$ of the serial diluent of tested drugs. Interpretation of results was performed after incubation at $35^{\circ} \mathrm{C}$ for $48 \mathrm{~h}$ for Fusarium spp. and $72 \mathrm{~h}$ for Exophiala spp., respectively. The MICs were determined as the lowest concentration resulting in complete inhibition of growth (Institute, 2008). All tests were performed in triplicate.

\section{In vitro Antifungal Susceptibility of Biofilms} The 96-well plate was washed with sterile PBS for three times to remove detached spores. The individual sessile minimum inhibitory concentrations (SMICs) of ITC, VRC, POS and AMB on photodynamic treated (T1) and untreated Exophiala and Fusarium biofilms were assessed by the XTT \{2,3-bis-(2-methoxy-4-nitro-5-sulfophenyl)-2H-tetrazolium-5carboxanilide based colorimetric assay (Ramage et al., 2001). After incubation at $37^{\circ} \mathrm{C}$ for $48 \mathrm{~h}, 100 \mu \mathrm{XTT} /$ menadione solution was added in each well and then incubated for another 4 h. Subsequently, $80 \mu \mathrm{l}$ of the colored supernatant from each well was removed and transferred into a new plate, and read at 490 $\mathrm{nm}$. The SMIC50 and SMIC80 was defined as the concentration at which a $50 \%$ or $80 \%$ decrease in optical density would be detected in comparison to the controls (Pierce et al., 2008). All tests were performed in triplicate.

\section{Analysis of Results}

For the purpose of analysis, CFU mL $\mathrm{m}^{-1}$ values were transformed into logarithm $\left(\log _{10}\right)$. The control group C1 was considered as $100 \%$ of growth for each set of tests. The photodynamic inactivation efficiency was evaluated by comparing the colony 
counts after treatments $\mathrm{T} 1, \mathrm{~T} 2$, and $\mathrm{T} 3$ with those obtained with non-irradiated and methylene blue-free control colonies (C1).

The effect of photodynamic inactivation on the antifungal susceptibility was evaluated by comparing the MICs and SMICs of the photodynamic treated (T1) planktonic cultures and biofilms with those untreated, respectively.

\section{RESULTS}

\section{Photodynamic Effects on the Growth of Planktonic Cultures and Biofilms}

The aPDT with methylene blue and LED exhibited CFU reductions of up to $3.8 \log _{10}$ and $6.4 \log _{10}$ against planktonic Exophiala spp. and Fusarium spp., respecitvely, and $4.2 \log _{10}$ and $5.6 \log _{10}$ against biofilms formed by Exophiala spp. and Fusarium spp., respecitvely, demonstrating efficacy in reducing the growth of both planktonic cultures and biofilms in all concentrations of methylene blue (Table 1 and Figures 1A-D).

\section{Photodynamic Effects on Antifungal Susceptibilities of Planktonic Cultures and Biofilms}

MIC ranges of four drugs against planktonic cells with or without aPDT were summarized in Table 2. Planktonic E. dermatitidis without aPDT showed MIC values of $1 \mu \mathrm{g} / \mathrm{ml}$ for ITC and AMB, and $0.25-0.5 \mu \mathrm{g} / \mathrm{ml}$ for VRC and POS (Table 2). MICs of planktonic E. dermatitidis decreased to $0.125 \mu \mathrm{g} / \mathrm{ml}$ for ITC and $0.06 \mu \mathrm{g} / \mathrm{ml}$ for VRC, POS and AMB, respectively, after aPDT treatment, as shown in Figure 2A. Planktonic culture of Fusarium spp. without PDT showed high MIC values of $\geq 16,4$ 8, 4-8, and 2-4 $\mu \mathrm{g} / \mathrm{ml}$ for ITC, VRC, POS and AMB, respectively (Table 2). However, after aPDT treatment, the MIC ranges decreased to $0.125-0.25,0.125,0.06-0.125$, and $0.06-0.125 \mu \mathrm{g} / \mathrm{ml}$ for ITC, VRC, POS, and AMB, respectively, as shown in Figure 2B.

SMIC ranges of four drugs against biofilms with or without PDT were summarized in Tables 3, 4. Fusarium and Exophiala biofilms showed high SMIC50 and SMIC80 of $\geq 16 \mu \mathrm{g} / \mathrm{ml}$ for all azoles tested. Both biofilms showed variable susceptibilities to $\mathrm{AMB}$, with SMIC ranging between 1 and $16 \mu \mathrm{g} / \mathrm{ml}$. Biofilms that were subjected to aPDT exhibited a distinct reduction in SMIC50 and SMIC80 compared to untreated groups for both species, except SMIC80 of ITC against Fusarium biofilms (Figures 2C-F).

\section{DISCUSSION}

Photodynamic inactivation combines the application of a pharmacologically inert chromophore, termed a photosensitizer (PS), and subsequent irradiation with visible light corresponding to the chromophore's specific absorption wavelength in the presence of molecular oxygen (Dai et al., 2012). After photon absorption the PS reaches an energized triplet state, which can undergo two mechanisms to regain its ground state. In type I mechanism, the PS directly transfers energy to a substrate or to molecular oxygen, producing reactive intermediates such as superoxide anion, hydrogen peroxide, hydroxyl radials, nitric oxide, and peroxide nitrite, while in type II mechanism, energy is transferred directly to molecular oxygen generating highly reactive singlet oxygen (Wainwright, 1998; Schweitzer and Schmidt, 2003; Hamblin and Hasan, 2004). The proportion of both mechanisms is unique for each PS with the singlet oxygen quantum yield $\Phi_{\Delta}$ describing the proportion of type II mechanism (Maisch et al., 2007).

A variety of PSs have been used in antifungal photodynamic inactivation, including toluidine blue, methylene blue, Rose Bengal, porphyrins, phthalocyanines, 5-aminolevulinic acid, and curcumin (Calzavara-Pinton et al., 2012; Baltazar et al., 2015). A newly developed photosensitizer SAPYR, which exhibits a singlet oxygen quantum yield of 0.99 and absorption wavelength of $360-$ $420 \mathrm{~nm}$, has been demonstrated stronger effect against bacterial biofilms than methylene blue that exhibits a singlet oxygen quantum yield of 0.52 (Cieplik et al., 2015). Phthalocyanines

TABLE 1 | Effect of photodynamic therapy on Exophiala spp. and Fusarium spp.

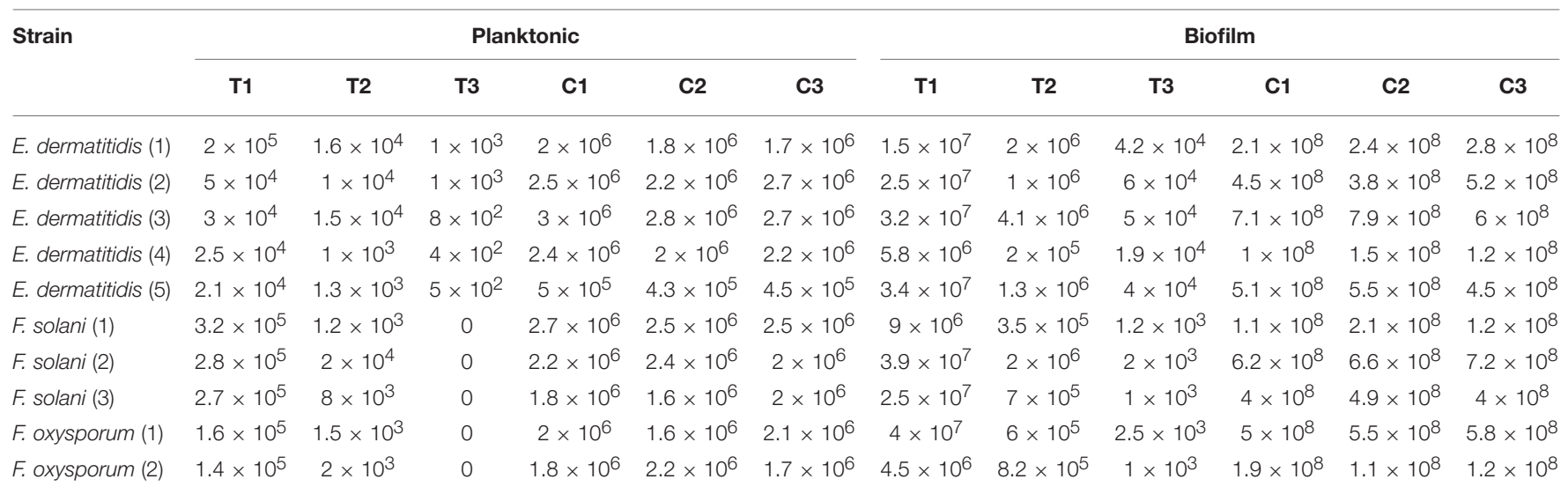

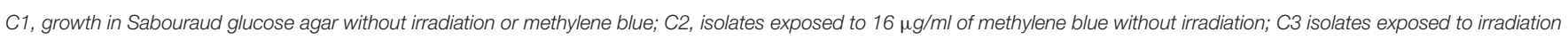

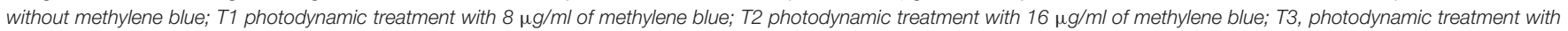
$32 \mu \mathrm{g} / \mathrm{ml}$ of methylene blue. 

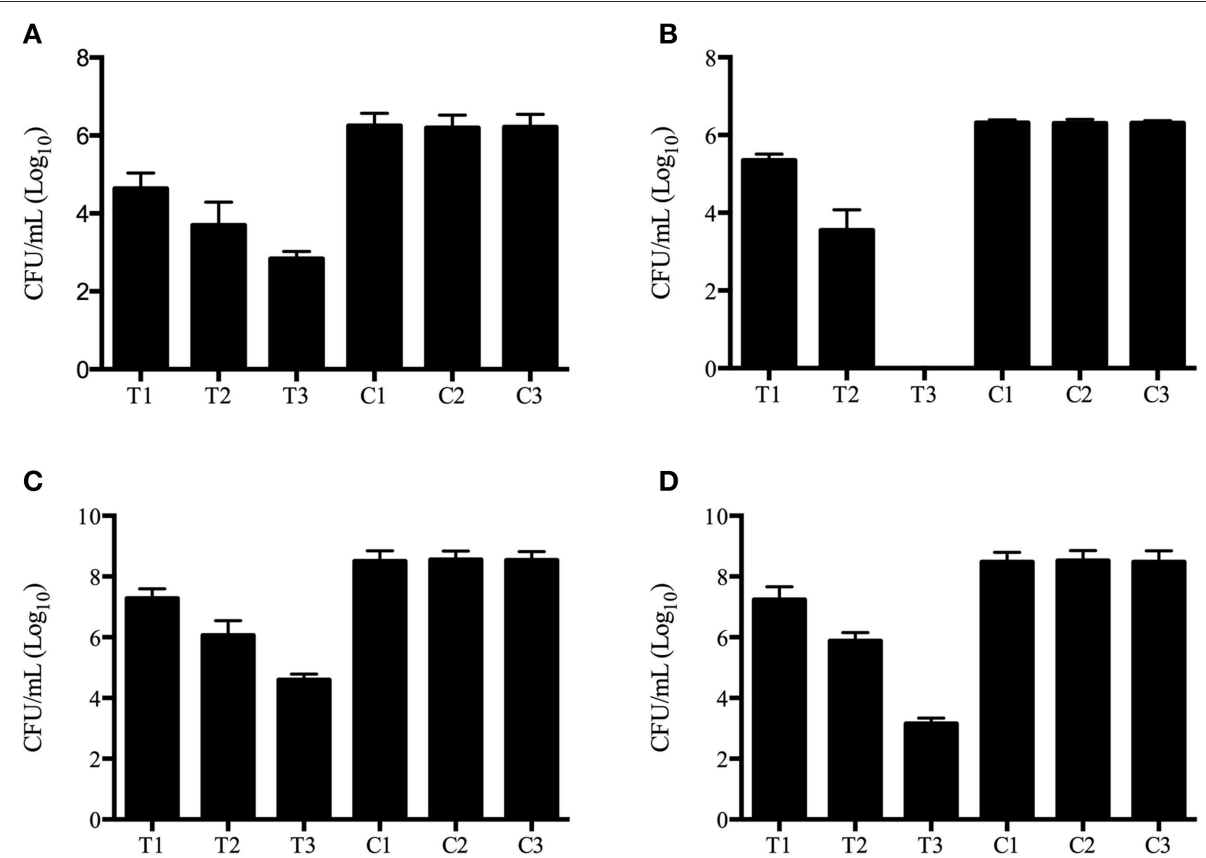

FIGURE 1 | Photodynamic inactivation effects on planktonic and biofilm forms of Exophiala spp. and Exophiala spp. (A) CFU counting of Planktonic Exophiala spp. (B) CFU counting of Planktonic Fusarium spp. (C) CFU counting of Exophiala Biofilms. (D) CFU counting of Fusarium Biofilms. C1, growth in Sabouraud glucose agar without irradiation or methylene blue; $\mathrm{C} 2$, isolates exposed to $16 \mu \mathrm{g} / \mathrm{ml}$ of methylene blue without irradiation; C3 isolates exposed to irradiation without methylene blue; T1 photodynamic treatment with $8 \mu \mathrm{g} / \mathrm{ml}$ of methylene blue; T2 photodynamic treatment with $16 \mu \mathrm{g} / \mathrm{ml}$ of methylene blue; T3, photodynamic treatment with $32 \mu \mathrm{g} / \mathrm{ml}$ of methylene blue. Data are mean values and standard error from three replicate experiments.

TABLE 2 | Effect of photodynamic therapy on MICs of planktonic culture.

\begin{tabular}{|c|c|c|c|c|c|c|c|c|}
\hline \multirow[t]{3}{*}{ Strain } & \multicolumn{8}{|c|}{ MICs $(\mu \mathrm{g} / \mathrm{ml})$} \\
\hline & \multicolumn{4}{|c|}{ Planktonic cultures } & \multicolumn{4}{|c|}{ Planktonic cultures with T1 treatment } \\
\hline & ITC & VRC & POS & AMB & ITC & VRC & POS & AMB \\
\hline E. dermatitidis (1) & 1 & 0.5 & 0.5 & 1 & 0.06 & 0.06 & 0.06 & 0.06 \\
\hline E. dermatitidis (2) & 1 & 0.25 & 0.5 & 1 & 0.06 & 0.06 & 0.06 & 0.06 \\
\hline E. dermatitidis (3) & 1 & 0.25 & 0.5 & 1 & 0.125 & 0.06 & 0.06 & 0.06 \\
\hline E. dermatitidis (4) & 1 & 0.25 & 0.5 & 1 & 0.06 & 0.06 & 0.06 & 0.06 \\
\hline E. dermatitidis (5) & 1 & 0.5 & 0.25 & 1 & 0.06 & 0.06 & 0.06 & 0.06 \\
\hline F. solani (1) & $\geq 16$ & 8 & 4 & 4 & 0.125 & 0.125 & 0.125 & 0.06 \\
\hline F. solani (2) & $\geq 16$ & 4 & 4 & 2 & 0.25 & 0.125 & 0.125 & 0.125 \\
\hline F. solani (3) & $\geq 16$ & 8 & 4 & 4 & 0.125 & 0.125 & 0.125 & 0.125 \\
\hline F. oxysporum (1) & $\geq 16$ & 8 & 4 & 2 & 0.25 & 0.125 & 0.06 & 0.06 \\
\hline F. oxysporum (2) & $\geq 16$ & 8 & 8 & 4 & 0.125 & 0.125 & 0.125 & 0.125 \\
\hline
\end{tabular}

are also characterized by high singlet oxygen quantum yields and high extinction coefficient in the far-red (680-720 nm) spectral region (Bertoloni et al., 1992; Calzavara-Pinton et al., 2012). A number of synthetic phthalocyanines, including chloroaluminum phthalocyanine and silicon phthalocyanine 4, were demonstrated effective in Candida albicans and Trichophyton rubrum in vitro (Lam et al., 2011, 2014; Carmello et al., 2016).

However, when choosing a PS for antifungal photodynamic inactivation, the light penetration is an important concern. Given that fungal infections involve not only the superficial skin, but also the subcutaneous tissue, nails, hair, nasal cavity, oral cavity, esophagus or reproductive tract, some degree of light penetration is required (Donnelly et al., 2008). Moreover, dematiaceous fungi possess pigments (melanin), which could interfere with light absorption. Therefore, for melanized fungi such as Exophiala spp., the PS selected should absorb light in a different wavelength from that of the pigment present in the fungi. It is important to note that methylene blue have a absorption wavelength over $600 \mathrm{~nm}$, which minimizes the competition with the melanin maximum absorption wavelength 

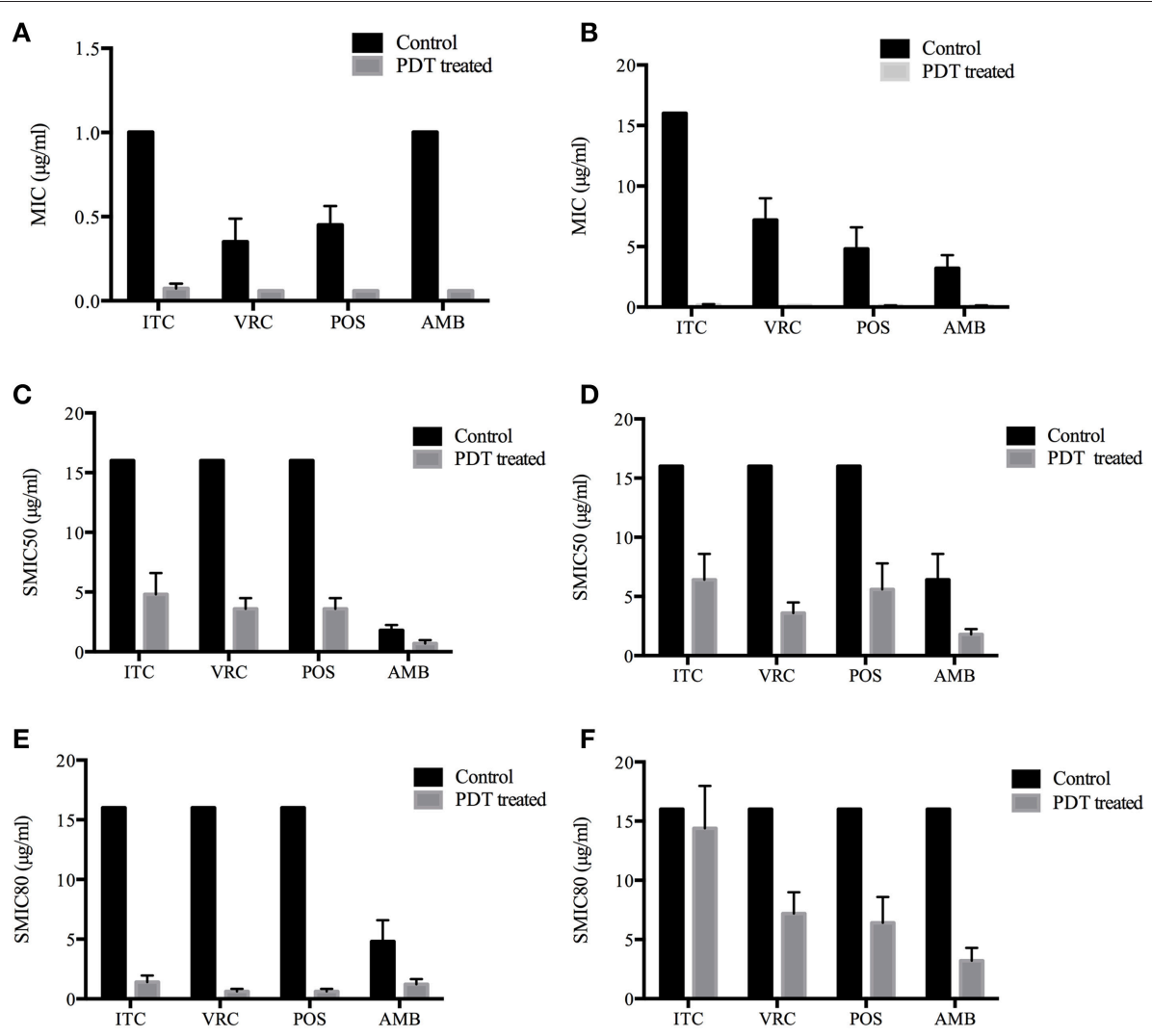

FIGURE 2 | Photodynamic effects on antifungal susceptibilities of planktonic and biofilm forms of Exophiala spp. and Exophiala spp. (A) MICs of Planktonic Exophiala spp. (B) MICs of Planktonic Fusarium spp. (C) SMIC50 of Exophiala biofilm. (D) SMIC50 of Fusarium biofilm. (E) SMIC80 of Exophiala biofilm. (F) SMIC80 of Fusarium biofilm. ITC, itraconazole; VRC, voriconazole; POS, posaconazole; AMB, amphotericin B. Data are mean values and standard error from three replicate experiments.

TABLE 3 | Effect of photodynamic therapy on SMIC50 of biofilms.

\begin{tabular}{|c|c|c|c|c|c|c|c|c|}
\hline \multirow[t]{3}{*}{ Strain } & \multicolumn{8}{|c|}{ SMIC50 $(\mu \mathrm{g} / \mathrm{ml})$} \\
\hline & \multicolumn{4}{|c|}{ Biofilm } & \multicolumn{4}{|c|}{$\begin{array}{l}\text { Biofilm with T1 } \\
\text { treatment }\end{array}$} \\
\hline & ITC & VRC & POS & AMB & ITC & VRC & POS & AMB \\
\hline E. dermatitidis (1) & $\geq 16$ & $\geq 16$ & $\geq 16$ & 2 & 4 & 4 & 4 & 0.5 \\
\hline E. dermatitidis (2) & $\geq 16$ & $\geq 16$ & $\geq 16$ & 1 & 4 & 4 & 2 & 0.5 \\
\hline E. dermatitidis (3) & $\geq 16$ & $\geq 16$ & $\geq 16$ & 2 & 8 & 4 & 4 & 0.5 \\
\hline E. dermatitidis (4) & $\geq 16$ & $\geq 16$ & $\geq 16$ & 2 & 4 & 2 & 4 & 1 \\
\hline E. dermatitidis (5) & $\geq 16$ & $\geq 16$ & $\geq 16$ & 2 & 4 & 4 & 4 & 1 \\
\hline F. solani (1) & $\geq 16$ & $\geq 16$ & $\geq 16$ & 4 & 4 & 4 & 8 & 2 \\
\hline F. solani (2) & $\geq 16$ & $\geq 16$ & $\geq 16$ & 8 & 8 & 4 & 4 & 2 \\
\hline F. solani (3) & $\geq 16$ & $\geq 16$ & $\geq 16$ & 4 & 8 & 4 & 4 & 1 \\
\hline F. oxysporum (1) & $\geq 16$ & $\geq 16$ & $\geq 16$ & 8 & 8 & 4 & 8 & 2 \\
\hline F. oxysporum (2) & $\geq 16$ & $\geq 16$ & $\geq 16$ & 8 & 4 & 2 & 4 & 2 \\
\hline
\end{tabular}

and allows maximal tissue transmission (Lyon et al., 2011a; Pires et al., 2014). Furthermore, the combination of methylene blue, which is already clinically approved for human use, and LED is a very inexpensive and convenient system, and is
TABLE 4 | Effect of photodynamic therapy on SMIC80 of biofilms.

\begin{tabular}{|c|c|c|c|c|c|c|c|c|}
\hline \multirow[t]{3}{*}{ Strain } & \multicolumn{8}{|c|}{ SMIC80 $(\mu \mathrm{g} / \mathrm{ml})$} \\
\hline & \multirow[b]{2}{*}{ ITC } & \multicolumn{2}{|c|}{ Biofilm } & \multicolumn{5}{|c|}{$\begin{array}{l}\text { Biofilm with } \mathrm{T} 1 \\
\text { treatment }\end{array}$} \\
\hline & & VRC & POS & AMB & ITC & VRC & POS & AMB \\
\hline E. dermatitidis (1) & $\geq 16$ & $\geq 16$ & $\geq 16$ & 8 & 2 & 0.5 & 0.5 & 2 \\
\hline E. dermatitidis (2) & $\geq 16$ & $\geq 16$ & $\geq 16$ & 4 & 1 & 0.5 & 0.5 & 1 \\
\hline E. dermatitidis (3) & $\geq 16$ & $\geq 16$ & $\geq 16$ & 4 & 2 & 1 & 1 & 1 \\
\hline E. dermatitidis (4) & $\geq 16$ & $\geq 16$ & $\geq 16$ & 4 & 1 & 0.5 & 0.5 & 1 \\
\hline E. dermatitidis (5) & $\geq 16$ & $\geq 16$ & $\geq 16$ & 4 & 1 & 0.5 & 0.5 & 1 \\
\hline F. solani (1) & $\geq 16$ & $\geq 16$ & $\geq 16$ & $\geq 16$ & 16 & 8 & 8 & 4 \\
\hline F. solani (2) & $\geq 16$ & $\geq 16$ & $\geq 16$ & $\geq 16$ & 8 & 4 & 4 & 2 \\
\hline F. solani (3) & $\geq 16$ & $\geq 16$ & $\geq 16$ & $\geq 16$ & 16 & 8 & 4 & 4 \\
\hline F. oxysporum (1) & $\geq 16$ & $\geq 16$ & $\geq 16$ & $\geq 16$ & 16 & 8 & 8 & 2 \\
\hline F. oxysporum (2) & $\geq 16$ & $\geq 16$ & $\geq 16$ & $\geq 16$ & 16 & 8 & 8 & 4 \\
\hline
\end{tabular}

increasingly being used in experimental and clinical applications of aPDT(Calzavara-Pinton et al., 2012; Dai et al., 2012). We therefore chose to apply aPDT using methylene blue and LED irradiation for studying the aPDT effects against planktonic and 
biofilm forms of Fusaruim spp. and Exophiala spp. in an in vitro assay.

The results in the present study revealed that all isolates tested were sensitive to photodynamic inactivation, both planktonic cells and biofilms. Planktonic and biofilm form of Exophiala spp. exhibited CFU reductions of up to $3.8 \log _{10}$ and 4.2 $\log _{10}$, respectively, which is declared as biologically relevant antimicrobial activity (Boyce et al., 2002). Planktonic and biofilm form of Fusarium spp. exhibited CFU reduction of up to $6.4 \log _{10}$ and $5.6 \log _{10}$, respectively, which is defined as disinfect effect (Boyce et al., 2002) and has not been reported in the literature thus far. Previous study has shown in C. albicans that ATP-binding cassette, a multidrug efflux system was directly implicated in methylene blue efflux from the cell cytoplasm, which might impact the antimicrobial photodynamic inactivation efficacy (Prates et al., 2011). However, in this study, Fusarium strains with high MIC values to ITC, VRC and POS were also sensitive to aPDT mediated by methylene blue. Despite Exophiala spp. showed relatively better antifungal susceptibility profile, the inactivation rates of planktonic and biofilm form of Fusaruim spp. in all treatment groups were comparable or even superior to Exophiala spp., suggesting that aPDT is active regardless of antifungal resistance, as described previously (Mima et al., 2010). This might relate to the principles of the action of nonspecific oxidizing agents of PDT, which oxidize biological molecules of the fungi cells in multiple targets (Lyon et al., 2011a).

Compared to planktonic cells, biofilms were less sensitive to aPDT since the irradiation time of biofilms was twice longer than that of planktonic cultures. Previously, biofilms have been found to be more resistant to aPDT than planktonic cells in several studies (Donnelly et al., 2007; Chabrier-Roselló et al., 2008; Dovigo et al., 2011; Costa et al., 2012). Therefore, longer preirradiation time or higher PS concentrations are needed to obtain better aPDT response (Donnelly et al., 2007; ChabrierRoselló et al., 2008; Dovigo et al., 2011; Costa et al., 2012). In this study, we were able to achieve satisfactory photodynamic effects by double the irradiation time of the biofilms. This difference probably occurred due to the structural characteristics of biofilms, including restriction of penetration by the extracellular matrix, the decreased growth rate and heterogeneity of the biofilm cells, and distinct gene expression levels (Costa et al., 2012).

We also tested the change of antifungal susceptibilities of tested strains before and after aPDT treatment, in both planktonic and biofilm forms. Pre-treatment with aPDT followed by standard antifungal treatments resulted in dramatic reduction

\section{REFERENCES}

Arboleda, A., Miller, D., Cabot, F., Taneja, M., Aguilar, M. C., Alawa, K., et al. (2014). Assessment of rose bengal versus riboflavin photodynamic therapy for inhibition of fungal keratitis isolates. Am. J. Ophthalmol. 158, 64-70.e62. doi: 10.1016/j.ajo.2014.04.007

Baltazar, L. M., Ray, A., Santos, D. A., Cisalpino, P. S., Friedman, A. J., and Nosanchuk, J. D. (2015). Antimicrobial photodynamic therapy: an effective of MICs and SMICs for both species. Both planktonic suspensions and biofilms were much more susceptible to antifungal drug treatments after aPDT, which may due to the increased membrane permeability caused by photodynamic inactivation, as demonstrated in previous study (Giroldo et al., 2009). The results suggest aPDT combined with standard antifungal treatment may help to enhance the antifungal susceptibility to overcome problems with drug resistance issues, and has the potential to reduce drug dosages and drug toxicity.

In general, our results expand the knowledge regarding the photodynamic inactivation of pathogenic fungi. The in vitro photodynamic therapy with methylene blue and LED was efficient in inhibiting the growth of Fusaruim spp. and Exophiala spp., both planktonic cultures and biofilms. In addition, the combination of aPDT and antifungal drugs represents an attractive alternative to the current antifungal strategies for infections of Fusaruim spp. and Exophiala spp., which has the potential to reduce treatment times, drug dosages, drug toxicity and improve patient compliance. However, despite the encouraging results, further investigations including in vivo experimental and clinical studies are warranted to determine clear protocols for the reliable and safe application in clinical practice.

\section{AUTHOR CONTRIBUTIONS}

LG, SJ, and YS conceived and designed the study. SJ, MD, and QW performed all the experiments. LG and YS analyzed the data and wrote the manuscript. ML and TZ provided general guidance and revised the manuscript.

\section{FUNDING}

This work was supported by grants 31400131 (LG) and 81401677 (YS) from National Natural Science Foundation of China, grant 2015ZSYXQN21 from Outstanding Youth Project of Zhongshan Hospital Fudan University (LG) and grant WJ2015MB281 from Hubei Province Health and Family Planning Scientific Research Project (YS).

\section{ACKNOWLEDGMENTS}

We thank Professor Ruoyu Li and Professor Wei Liu from Peking University First Hospital, Research Center for Medical Mycology, Peking University, Beijing, and Professor Qiangqiang Zhang from Fudan University Huashan Hospital, Shanghai, for kindly provided us with isolates studied. prokaryotic and eukaryotic microbial cells. Microbios 71, 33-46.

Boyce, J. M., Pittet, D., Healthcare Infection Control Practices Advisory Committee and HICPAC/SHEA/APIC/IDSA Hand Hygiene Task Force. (2002). Guideline for hand hygiene in health-care settings: recommendations 
of the Healthcare Infection Control Practices Advisory Committee and the HICPAC/SHEA/APIC/IDSA Hand Hygiene Task Force. Infect. Control Hosp. Epidemiol. 23, S3-S40. doi: 10.1086/503164

Calzavara-Pinton, P., Rossi, M. T., Sala, R., and Venturini, M. (2012). Photodynamic antifungal chemotherapy. Photochem. Photobiol. 88, 512-522. doi: 10.1111/j.1751-1097.2012.01107.x

Carmello, J. C., Alves, F., Ribeiro, A. P. D., Basso, F. G., de Souza Costa, C. A., Tedesco, A. C., et al. (2016). In vivo photodynamic inactivation of Candida albicans using chloro-aluminum phthalocyanine. Oral Dis. doi: 10.1111/odi.12466. [Epub ahead of print].

Chabrier-Roselló, Y., Foster, T. H., Mitra, S., and Haidaris, C. G. (2008). Respiratory deficiency enhances the sensitivity of the pathogenic fungus Candida to photodynamic treatment. Photochem. Photobiol. 84, 1141-1148. doi: 10.1111/j.1751-1097.2007.00280.x

Cieplik, F., Pummer, A., Regensburger, J., Hiller, K. A., Späth, A., Tabenski, L., et al. (2015). The impact of absorbed photons on antimicrobial photodynamic efficacy. Front. Microbiol. 6:706. doi: 10.3389/fmicb.2015.00706

Costa, A. C., Rasteiro, V. M., Pereira, C. A., Rossoni, R. D., Junqueira, J. C., and Jorge, A. O. (2012). The effects of rose bengal- and erythrosinemediated photodynamic therapy on Candida albicans. Mycoses 55, 56-63. doi: 10.1111/j.1439-0507.2011.02042.x

Dai, T., Fuchs, B. B., Coleman, J. J., Prates, R. A., Astrakas, C., St. Denis, T. G., et al. (2012). Concepts and principles of photodynamic therapy as an alternative antifungal discovery platform. Front. Microbiol. 3:120. doi: 10.3389/fmicb.2012.00120

Davies, D. (2003). Understanding biofilm resistance to antibacterial agents. Nat. Rev. Drug Discov. 2, 114-122. doi: 10.1038/nrd1008

Desai, J. V., Mitchell, A. P., and Andes, D. R. (2014). Fungal biofilms, drug resistance, and recurrent infection. Cold Spring Harb. Perspect. Med. 4:a019729. doi: 10.1101/cshperspect.a019729

Donnelly, R. F., McCarron, P. A., and Tunney, M. M. (2008). Antifungal photodynamic therapy. Microbiol. Res. 163, 1-12. doi: 10.1016/j.micres.2007.08.001

Donnelly, R. F., McCarron, P. A., Tunney, M. M., and David Woolfson, A. (2007). Potential of photodynamic therapy in treatment of fungal infections of the mouth. Design and characterisation of a mucoadhesive patch containing toluidine blue O. J. Photochem. Photobiol. B 86, 59-69. doi: 10.1016/j.jphotobiol.2006.07.011

Dovigo, L. N., Pavarina, A. C., Ribeiro, A. P., Brunetti, I. L., Costa, C. A., Jacomassi, D. P., et al. (2011). Investigation of the photodynamic effects of curcumin against Candida albicans. Photochem. Photobiol. 87, 895-903. doi: 10.1111/j.1751-1097.2011.00937.x

Filizzola, M. J., Martinez, F., and Rauf, S. J. (2003). Phaeohyphomycosis of the central nervous system in immunocompetent hosts: report of a case and review of the literature. Int. J. Infect. Dis. 7, 282-286. doi: 10.1016/S12019712(03)90108-1

Gilaberte, Y., Aspiroz, C., Martes, M. P., Alcalde, V., Espinel-Ingroff, A., and Rezusta, A. (2011). Treatment of refractory fingernail onychomycosis caused by nondermatophyte molds with methylaminolevulinate photodynamic therapy. J. Am. Acad. Dermatol. 65, 669-671. doi: 10.1016/j.jaad.2010.06.008

Giroldo, L. M., Felipe, M. P., de Oliveira, M. A., Munin, E., Alves, L. P., and Costa, M. S. (2009). Photodynamic antimicrobial chemotherapy (PACT) with methylene blue increases membrane permeability in Candida albicans. Lasers Med. Sci. 24, 109-112. doi: 10.1007/s10103-007-0530-2

Guarro, J. (2013). Fusariosis, a complex infection caused by a high diversity of fungal species refractory to treatment. Eur. J. Clin. Microbiol. Infect. Dis. 32, 1491-1500. doi: 10.1007/s10096-013-1924-7

Hamblin, M. R., and Hasan, T. (2004). Photodynamic therapy: a new antimicrobial approach to infectious disease? Photochem. Photobiol. Sci. 3, 436-450. doi: $10.1039 / \mathrm{b} 311900 \mathrm{a}$

Institute, C.a.L.S. (2008). Reference Method for Broth Dilution Antifungal Susceptibility Testing of Filamentous Fungi. Approved Standard M38-A2. Wayne, PA:CLSI.

Kashiwabuchi, R. T., Carvalho, F. R., Khan, Y. A., Hirai, F., Campos, M. S., and McDonnell, P. J. (2013). Assessment of fungal viability after long-wave ultraviolet light irradiation combined with riboflavin administration. Graefes Arch. Clin. Exp. Ophthalmol. 251, 521-527. doi: 10.1007/s00417-012-2209-z
Kondori, N., Gilljam, M., Lindblad, A., Jönsson, B., Moore, E. R., and Wennerås, C. (2011). High rate of Exophiala dermatitidis recovery in the airways of patients with cystic fibrosis is associated with pancreatic insufficiency. J. Clin. Microbiol. 49, 1004-1009. doi: 10.1128/JCM.01899-10

Lam, M., Dimaano, M. L., Oyetakin-White, P., Retuerto, M. A., Chandra, J., Mukherjee, P. K., et al. (2014). Silicon phthalocyanine 4 phototoxicity in Trichophyton rubrum. Antimicrob. Agents Chemother. 58, 3029-3034. doi: 10.1128/AAC.01448-13

Lam, M., Jou, P. C., Lattif, A. A., Lee, Y., Malbasa, C. L., Mukherjee, P. K., et al. (2011). Photodynamic therapy with Pc 4 induces apoptosis of Candida albicans. Photochem. Photobiol. 87, 904-909. doi: 10.1111/j.1751-1097.2011. 00938.x

Li, D. M., Li, R. Y., de Hoog, G. S., Sudhadham, M., and Wang, D. L. (2011). Fatal Exophiala infections in China, with a report of seven cases. Mycoses 54, e136-e142. doi: 10.1111/j.1439-0507.2010.01859.x

Lyon, J. P., Moreira, L. M., de Carvalho, V. S., dos Santos, F. V., de Lima, C. J., and de Resende, M. A. (2013). In vitro photodynamic therapy against Foncecaea pedrosoi and Cladophialophora carrionii. Mycoses 56, 157-161. doi: 10.1111/j.1439-0507.2012.02226.x

Lyon, J. P., Moreira, L. M., de Moraes, P. C., dos Santos, F. V., and de Resende, M. A. (2011a). Photodynamic therapy for pathogenic fungi. Mycoses 54, e265e271. doi: 10.1111/j.1439-0507.2010.01966.x

Lyon, J. P., Pedroso e Silva Azevedo Cde, M., Moreira, L. M., de Lima, C. J., and de Resende, M. A. (2011b). Photodynamic antifungal therapy against chromoblastomycosis. Mycopathologia 172, 293-297. doi: 10.1007/s11046-0119434-6

Maisch, T., Baier, J., Franz, B., Maier, M., Landthaler, M., Szeimies, R. M., et al. (2007). The role of singlet oxygen and oxygen concentration in photodynamic inactivation of bacteria. Proc. Natl. Acad. Sci. U.S.A. 104, 7223-7228. doi: 10.1073/pnas.0611328104

Mima, E. G., Pavarina, A. C., Dovigo, L. N., Vergani, C. E., Costa, C. A., Kurachi, C., et al. (2010). Susceptibility of Candida albicans to photodynamic therapy in a murine model of oral candidosis. Oral Surg. Oral Med. Oral Pathol. Oral Radiol. Endod. 109, 392-401. doi: 10.1016/j.tripleo.2009.10.006

Morton, C. O., Chau, M., and Stack, C. (2014). In vitro combination therapy using low dose clotrimazole and photodynamic therapy leads to enhanced killing of the dermatophyte Trichophyton rubrum. BMC Microbiol. 14:261. doi: 10.1186/s12866-014-0261-z

Nunes Mario, D. A., Denardi, L. B., Brayer Pereira, D. I., Santurio, J. M., and Alves, S. H. (2014). In vitro photodynamic inactivation of Sporothrix schenckii complex species. Med. Mycol. 52, 770-773. doi: 10.1093/mmy/myu041

Patel, A. K., Patel, K. K., Darji, P., Singh, R., Shivaprakash, M. R., and Chakrabarti, A. (2013). Exophiala dermatitidis endocarditis on native aortic valve in a postrenal transplant patient and review of literature on E. dermatitidis infections. Mycoses 56, 365-372. doi: 10.1111/myc. 12009

Pereira, C. A., Romeiro, R. L., Costa, A. C., Machado, A. K., Junqueira, J. C., and Jorge, A. O. (2011). Susceptibility of Candida albicans, Staphylococcus aureus, and Streptococcus mutans biofilms to photodynamic inactivation: an in vitro study. Lasers Med. Sci. 26, 341-348. doi: 10.1007/s10103-010-0852-3

Pereira Gonzales, F., and Maisch, T. (2012). Photodynamic inactivation for controlling Candida albicans infections. Fungal Biol. 116, 1-10. doi: 10.1016/j.funbio.2011.10.001

Pierce, C. G., Uppuluri, P., Tristan, A. R., Wormley, F. L. Jr., Mowat, E., Ramage, G., et al. (2008). A simple and reproducible 96-well plate-based method for the formation of fungal biofilms and its application to antifungal susceptibility testing. Nat. Protoc. 3, 1494-1500. doi: 10.1038/nprot.2008.141

Pires, L., Bosco Sde, M., Baptista, M. S., and Kurachi, C. (2014). Photodynamic therapy in Pythium insidiosum - an in vitro study of the correlation of sensitizer localization and cell death. PLoS ONE 9:e85431. doi: 10.1371/journal.pone.0085431

Pires, L., Bosco Sde, M., da Silva, N. F. Jr., and Kurachi, C. (2013). Photodynamic therapy for pythiosis. Vet. Dermatol. 24, 130-136.e130. doi: 10.1002/9781118644317.ch18

Prates, R. A., Kato, I. T., Ribeiro, M. S., Tegos, G. P., and Hamblin, M. R. (2011). Influence of multidrug efflux systems on methylene blue-mediated photodynamic inactivation of Candida albicans. J. Antimicrob. Chemother. 66, 1525-1532. doi: 10.1093/jac/dkr160 
Ramage, G., Vande Walle, K., Wickes, B. L., and López-Ribot, J. L. (2001). Standardized method for in vitro antifungal susceptibility testing of Candida albicans biofilms. Antimicrob. Agents Chemother. 45, 2475-2479. doi: 10.1128/AAC.45.9.2475-2479.2001

Revankar, S. G., and Sutton, D. A. (2010). Melanized fungi in human disease. Clin. Microbiol. Rev. 23, 884-928. doi: 10.1128/CMR.00019-10

Sauer, A., Letscher-Bru, V., Speeg-Schatz, C., Touboul, D., Colin, J., Candolfi, E., et al. (2010). In vitro efficacy of antifungal treatment using riboflavin/UV-A (365 nm) combination and amphotericin B. Invest. Ophthalmol. Vis. Sci. 51, 3950-3953. doi: 10.1167/iovs.09-4013

Schweitzer, C., and Schmidt, R. (2003). Physical mechanisms of generation and deactivation of singlet oxygen. Chem. Rev. 103, 1685-1757. doi: $10.1021 / \mathrm{cr} 010371 \mathrm{~d}$

Souza, L. W., Souza, S. V., and Botelho, A. C. (2014). Distal and lateral toenail onychomycosis caused by Trichophyton rubrum: treatment with photodynamic therapy based on methylene blue dye. An. Bras. Dermatol. 89, 184-186. doi: 10.1590/abd1806-4841.20142197

Wainwright, M. (1998). Photodynamic antimicrobial chemotherapy (PACT). J. Antimicrob. Chemother. 42, 13-28. doi: 10.1093/jac/42.1.13

Conflict of Interest Statement: The authors declare that the research was conducted in the absence of any commercial or financial relationships that could be construed as a potential conflict of interest.

Copyright (c) 2016 Gao, Jiang, Sun, Deng, Wu, Li and Zeng. This is an open-access article distributed under the terms of the Creative Commons Attribution License (CC $B Y)$. The use, distribution or reproduction in other forums is permitted, provided the original author(s) or licensor are credited and that the original publication in this journal is cited, in accordance with accepted academic practice. No use, distribution or reproduction is permitted which does not comply with these terms. 